\title{
Relationship between DIAGNOdent values and sealant penetration depth on occlusal fissures
}

\author{
Jonas A. Rodrigues • Michele B. Diniz • Isabel Hug • \\ Rita C. L. Cordeiro • Adrian Lussi
}

Received: 3 March 2009 /Accepted: 21 July 2009 /Published online: 5 August 2009

(C) Springer-Verlag 2009

\begin{abstract}
The aim of this in vitro study was to evaluate the relationship between laser fluorescence values and sealant penetration depth on occlusal fissures. One hundred and sixty-six permanent molars were selected and divided into four groups, which were each treated using a different sealant (two clear and two opaque). The teeth were independently measured twice by two experienced dentists using two laser fluorescence devices-DIAGNOdent (LF and LFpen) - before and after sealing, and then thermoclycled. After measuring, the teeth were histologically prepared and assessed for caries extension. Digital photographs of the cut sealed sites were assessed, and the sealant penetration depth was measured. All 166 sites were measured by one of the examiners taking as limits the outer and inner surface of the sealant into the fissure. For each device (LF and LFpen) and each group, the difference between the values at baseline and after sealing was plotted against the sealant penetration depth and scatter plots were provided. It could be observed that most of the points were concentrated around the zero line, for both LF and LFpen in the four groups. In conclusion, there is no relation between changes in DIAGNOdent values and increasing of depth sealant penetration within the occlusal fissures.
\end{abstract}

J. A. Rodrigues $(\bowtie) \cdot$ I. Hug $\cdot$ A. Lussi

Department of Preventive, Restorative and Pediatric Dentistry,

School of Dental Medicine, University of Bern,

Freiburgstrasse 7,

CH 3010 Bern, Switzerland

e-mail: jonas.rodrigues@zmk.unibe.ch

M. B. Diniz $\cdot$ R. C. L. Cordeiro

Department of Pediatric Dentistry,

Araraquara School of Dentistry, UNESP,

Rua Humaitá, 1680 CEP:14801-903, Araraquara,

São Paulo, Brazil
Keywords Dental caries · Detection · DIAGNOdent . Laser fluorescence $\cdot$ Occlusal sealants $\cdot$ Teeth

\section{Introduction}

Occlusal surfaces are particularly vulnerable to caries development due to their morphological complexity, which favour plaque accumulation and difficult fluoride exposure [1]. The application of pit and fissure sealants has shown to be an effective method to prevent occlusal caries $[1,2]$ and to cease incipient caries activity $[3,4]$. However, the presence of sealants influences the performance of conventional methods in detecting occlusal caries and in monitoring its progression. Thus, adjunct methods must be used to improve the monitoring assessments and to increase the diagnostic accuracy [5].

Some of these methods are based on the fluorescence, which is emitted by the porphyrins present in caries lesions when stimulated at specific excitation wavelengths $[6,7]$. Two laser fluorescence devices DIAGNOdent 2095 (LF; KaVo, Biberach, Germany) and DIAGNOdent 2190 (LFpen; KaVo, Biberach, Germany) have been currently used as adjunct in caries detection. Several studies have shown good results of validity and reproducibility on occlusal surfaces concerning both of these devices [8-12], although a systematic review of the literature addressed that its usefulness as a diagnostic tool is limited [7]. Nevertheless, the presence of sealants and composite filling materials might complicate the interpretation of the LF readings leading to false-positive results $[5,13,14]$.

There are few studies concerning the influence of pit and fissure sealants on fluorescence measurements. It is important to stress that there are differences between opaque and clear sealants on the fluorescence readings. 
Previous studies have shown that fissure sealants do not influence LF readings and suggested that the LF device could be used to monitor and to detect caries under fissure sealants [15-18]. However, other studies have shown opposite results [14, 19]. So far, only one study has investigated the performance of LFpen comparing with LF to detect caries under different pit and fissure sealants [5]. The authors concluded that both laser fluorescence devices could be useful as an adjunct to detect occlusal caries under unfilled clear sealants.

However, it has been suggested that the depth of the sealant penetration into pit and fissures could influence the fluorescence readings. The fissure type and anatomy play a significant role on the sealant amount that would penetrate into fissures [20]. Therefore, it could be expected that there might be a significant correlation between fluorescence measurements and the depth of penetration of the sealant. For this reason, the authors of the present investigation proposed to continue the analysis of the sample of the previously published study [5] with the aim of evaluating the relationship between DIAGNOdent values and sealant penetration depth on occlusal fissures.

\section{Materials and methods}

The sample of a previously published study [5], which is composed of the 166 third permanent human molars extracted, were used. Teeth were stored frozen at $-20^{\circ} \mathrm{C}$ until use and during the experiments. Earlier studies showed that this method of storage does not change the red fluorescence significantly [21]. All teeth were extracted by dental practitioners in Switzerland (no water fluoridation, $250 \mathrm{ppm} \mathrm{F}^{-}$in table salt). Prior to extraction, the patients were informed about the use of their teeth for research purposes and their written consent was obtained. The teeth were defrosted for $3 \mathrm{~h}$, and the calculus and debris were removed using a scaler (Cavitron). They were cleaned for $15 \mathrm{~s}$ with water and toothbrush (Trisa ultra super-sensitive; Triengen, Switzerland) and for $10 \mathrm{~s}$ with a water-powder jet cleaner (PROPHYflex II; KaVo, Biberach, Germany) and sodium hydrogen carbonate powder. In order not to have powder remnants in the fissure, the teeth were then rinsed off with a 3-in-1 syringe for $10 \mathrm{~s}$ [11].

Photographs of the occlusal surfaces were taken at a $\times 6.25$ magnification using a light microscope (Leica DC300; Leica, Heerbrugg, Switzerland) equipped with a video camera linked to a computer (Leica M420; Leica, Heerbrugg, Switzerland), and the test site was marked. All assessments were independently carried out twice by two experienced dentists, with a 1-week interval between the measurements. The following devices were used: DIAGNOdent 2095 (LF), DIAGNOdent 2190 (LFpen; both from
Kavo, Biberach, Germany). During the measurements, the teeth were stored in $100 \%$ humidity. Measurements were made before (baseline) and after sealing.

\section{Baseline measurements}

Measurements were performed with LF using the probe tip " $\mathrm{A}$ " and with the LFpen using the cylindrical sapphire fibre tip, according to manufacturer's instructions. The manufacturer recommends that the device should be calibrated before each patient or when peak value is \pm 3 of standard when checked against the ceramic standard. As the present investigation was performed in in vitro conditions, with teeth collected from different patients, the device was calibrated for each tooth. Afterwards, the zero value of fluorescence of a sound part of the cuspal area on the buccal surface was recorded. The tip was placed on the selected site and rotated around the vertical axis until the highest fluorescence reading was obtained. The peak values were recorded and the zero value of fluorescence was subtracted.

After measuring, in order to divide the teeth into groups, the average fluorescence values obtained with the LF device and the cut-off limits suggested by Lussi and Hellwig (2006) were used to form groups with the same number of sound teeth, enamel and dentine caries. The teeth were then randomly divided according to the material for pit and fissure sealing. The groups were arranged as follows:

- Group I - Delton Unfilled Clear (Dentsply, Konstanz, Germany); 41 teeth;

- Group II - Delton Opaque (Dentsply, Konstanz, Germany); 42 teeth;

- Group III - Helioseal Opaque (Ivoclar Vivadent, Schaan, Liechtenstein); 41 teeth;

- Group IV - Experimental Nanofilled Clear (Voco, Cushaven, Germany). 42 teeth.

\section{Sealant placement}

Occlusal surfaces were etched with $35 \%$ phosphoric acid gel (Vococid etching liquid; Voco, Cuxhaven, Germany) for $60 \mathrm{~s}$. The etchant was gently stirred on the occlusal surfaces using a soft microbrush. Teeth were then rinsed with water/ air spray for $15 \mathrm{~s}$ and dried with an air syringe for $5 \mathrm{~s}$. The sealant was applied directly onto the etched and dried surface with a round-ended applicator BR 06/08 (A. Deppeler SA, Rolle, Switzerland), taking care not to overfill the fissures and avoiding contact of the applicator with the enamel surfaces. The sealant was left undisturbed for $20 \mathrm{~s}$ in order to allow it to flow into the fissures system and over the etched surface. After that, the sealant was light 
cured for $40 \mathrm{~s}$ (Optilux 400, $300 \mathrm{~mW} / \mathrm{cm}^{2}$; Demetron Reseacrh Corp.) [20]. Photographs at a magnification of $\times 6.25$ were taken.

After sealing, the same examiners remeasured the teeth using the fluorescence-based devices as described previously. The sealed teeth were thermocycled in deionized water for 1,000 cycles between $5 \pm 2$ and $55 \pm 2^{\circ} \mathrm{C}$, with a dwell time of $30 \mathrm{~s}$.

\section{Histological preparation}

The teeth were ground longitudinally on a rotating polishing machine (Knuth-Rotor; Struers, Copenhagen, Denmark) using a silicon carbide paper $(60-\mu \mathrm{m}$ grain size $)$ cooled under tap water. Progress of grinding was constantly checked under the microscope (magnification $\times 6.25$ ) and compared with the initial pictures of the test sites. When the periphery of the site was reached, papers of grain size 30 , 18,8 and $5 \mu \mathrm{m}$ were used and the occlusal cut sealed sites were then photographed (Leica DC300; Leica, Heerbrugg, Switzerland).

\section{Depth measurements}

Digital photographs of the cut sealed sites were assessed using the software IM500 version 4.0 (Leica, Cambridge, UK), and the sealant penetration depth was measured. All 166 sites were measured by one of the examiners taking as limits the outer and inner surface of the sealant into the fissure.

\section{Statistical analysis}

The average of the four measurements was obtained for each tooth at baseline and after sealing. For each device (LF and LFpen) and each group, the difference between the values at baseline and after sealing was plotted against the sealant penetration depth and scatter plots were provided (Fig. 1).

\section{Results}

The scatter plots (Fig. 1) give an overview of the distribution of the difference in laser fluorescence values comparing before and after sealing according to the sealant penetration depth. In general, it could be observed that most of the data points were closer around the zero line, for both LF and LFpen for the four groups, indicating no correlation between the fluorescence values and the sealant penetration depth. For clear sealants, it could be observed that the differences between the fluorescence values ranged up to 20 units. For group I, the differences in fluorescence varied from negative to positive values independent of the sealant depth penetration. For group IV, most of the points were observed in the positive area of the graphic, mainly between 0.5 and $1.0 \mathrm{~mm}$ of sealant depth penetration.

However, for both opaque sealants (groups II and III), it could be observed that the differences between the fluorescence values were negative and ranged up to 20 and up to 30 units, respectively. For group II, this difference was observed into the interval between 0.5 and $1.0 \mathrm{~mm}$ of sealant depth penetration, whereas for group III, between 0.0 and $1.0 \mathrm{~mm}$.

\section{Discussion}

Although pit and fissure sealants have been shown to be effective for caries prevention, sealed surfaces require careful assessment and monitoring for caries detection since the examiners can underestimate the severity of lesions detected after sealing compared with their assessment prior to sealing [22]. As occlusal fissures show different anatomic characteristics [23, 24], the sealants penetration pattern can vary and might become a barrier for caries diagnosis. Thus, adjunct methods must be used to improve the follow-up assessments and to increase the diagnostic accuracy [5].

In a previous published study [5], the authors evaluated the influence of different pit and fissures sealants on fluorescence values and found that sealants influence fluorescence readings and that LF and LFpen could be useful as adjunct to detect occlusal caries under unfilled clear sealants. The present study is a continuation in which the relationship between the depth of penetration of different occlusal sealants and fluorescence values were evaluated.

In the presented study, when the difference between the LF values before and after sealing were plotted against the sealant penetration depth, most of the points ranged around the zero line even when the depth increased. The clear materials used in this set up showed some cases in which the difference in the fluorescence values was up to 20 units. For group I, positive differences mean that the fluorescence values increased after sealing, and negative differences mean that the values decreased. According to Diniz et al. (2008), fluorescence readings of the Delton Clear sealant increased in thicker samples. However, they only evaluated sealant specimens, and in the present investigation, occlusal sound and carious sealed surfaces were evaluated.

For group IV, the differences were positive, indicating that a tendency of values increasing was observed after sealing. In the previous set up [5], a tendency of values increasing was described as well. The authors attributed the values increasing to the etching time that modify the 
Fig. 1 Scatter plots showing the difference between the fluorescence values at baseline and after sealing for each device (LF and LFpen) and each group plotted against the sealant penetration depth
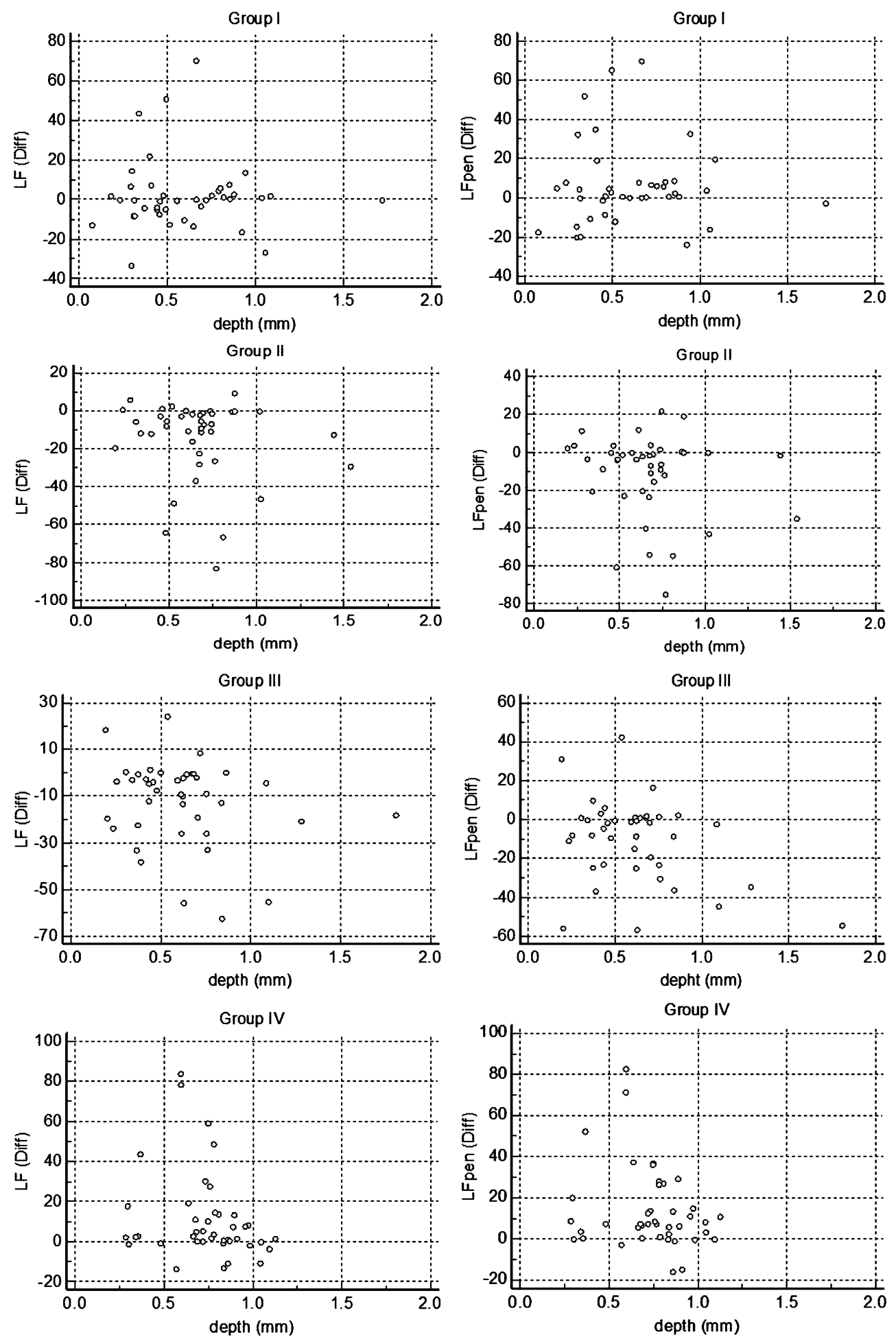

scattering properties of the treated enamel and to the translucency of the material, which allow the light to reach the etched surface. The results of the present study support this statement, where no relation between LF values, and the increasing of the sealed fissure depth was observed.

For the opaque sealants, some differences, up to 30 units, could be observed independently of the fissure depth increasing. As those differences were negative, this means that the fluorescence values decreased after sealing. This shows that the fissure anatomy and, consequently, the amount of sealant that penetrates within the fissure are not related to the LF values decreasing. It has already been shown by Diniz et al. [5] that LF values decrease significantly after sealing using opaque materials (Helioseal 
and Delton) and a decrease of 20 units of fluorescence can represent a false-negative diagnostic, according to the cutoff limits suggested in the literature. This change in the values is hence not due to the amount of sealant that penetrates into the fissure but to the opacifiers, which present an intrinsic fluorescence. Furthermore, the variation found in a sound site could not be the same as the variation found for a dentin caries lesion, because the LF values are, obviously, not the same. Outlier values could also be an explanation. Our results show that opaque sealants influence the fluorescence readings for both devices, although there is no correlation with the sealant penetration depth.

Thermocycling was performed to simulate the thermal stressing between the tooth and dental materials, which normally occurs in the mouth with the ingestion of hot and cold liquids. From the pictures, color changes and loss of marginal integrity could be observed. For this reason, the depth of sealant penetration was measured after thermocycling.

Despite our results, the clinicians need to take care not to overfill the fissure, as successive thermal stresses may lead to a higher rate of marginal failure and microleakage in overfilled fissures [20]. Besides, some studies have shown that unfilled sealant penetrates more deeply into the fissures and is better retained [4, 25]. In agreement with Diniz et al. [5], unfilled clear fissure sealants could allow better detection of the occlusal lesions using the LF device, independently of the sealant penetration depth.

In conclusion, there is no relation between changes in DIAGNOdent values and increasing penetration depth of sealant within the occlusal fissures.

Conflict of interest The authors declare that they have no conflict of interest.

\section{References}

1. Manton DJ, Messer LB (1995) Pit and fissure sealants: another major cornerstone in preventive dentistry. Aust Dent J 40:20-29

2. Simonsen RJ (1991) Retention and effectiveness of dental sealant after 15 years. J Am Dent Assoc 122:34-42

3. Mertz-Fairhurst EJ, Curtis JW Jr, Ergle JW, Rueggeberg FA, Adair SM (1998) Ultraconservative and cariostatic sealed restorations: results at year 10. J Am Dent Assoc 129:55-66

4. Simonsen RJ (2002) Pit and fissure sealant: review of the literature. Pediatr Dent 24:393-414

5. Diniz MB, Rodrigues JA, Hug I, Cordeiro RC, Lussi A (2008) The influence of pit and fissure sealants on infrared fluorescence measurements. Caries Res 42:328-333

6. Hibst R, Paulus R, Lussi A (2001) A detection of occlusal caries by laser fluorescence: basic and clinical investigations. Med Laser Appl 16:295-213
7. Bader JD, Shugars DA (2004) A systematic review of the performance of a laser fluorescence device for detecting caries. J Am Dent Assoc 135:1413-1426

8. Rodrigues JA, Hug I, Diniz MB, Lussi A (2008) Performance of fluorescence methods, radiographic examination and ICDAS II on occlusal surfaces in vitro. Caries Res 42:297-304

9. Sheehy EC, Brailsford SR, Kidd EAM, Beighton D, Zoitopoulos L (2001) Comparison between visual examination and a laser fluorescence system for in vivo diagnosis of occlusal caries. Caries Res 35:421-426

10. Lussi A, Imwinkelried S, Pitts N, Longbottom C, Reich E (1999) Performance and reproducibility of a laser fluorescence system for detection of occlusal caries in vitro. Caries Res 33:261-266

11. Lussi A, Hellwig E (2006) Performance of a new laser fluorescence device for the detection of occlusal caries in vitro. J Dent 34:467-471

12. Diniz MB, Rodrigues JD, Paula AB, Cordeiro RD (2009) In vivo evaluation of laser fluorescence performance using different cut-off limits for occlusal caries detection. Lasers Med Sci 24:295-300

13. Lussi A, Reich E (2005) The influence of toothpastes and prophylaxis pastes on fluorescence measurements for caries detection in vitro. Eur J Oral Sci 113:141-144

14. Hosoya Y, Matsuzaka K, Inoue T, GW M (2004) Influence of tooth-polishing pastes and sealants on DIAGNOdent values. Quintessence Int 35:605-611

15. Anttonen V, Seppä L, Hausen H (2003) Clinical study of the use of the laser fluorescence device DIAGNOdent for detection of occlusal caries in children. Caries Res 37:17-23

16. Deery C, Iloya J, Nugent ZJ, Srinivasan V (2006) Effect of placing a clear sealant on the validity and reproducibility of occlusal caries detection by a laser fluorescence device: An in vitro study. Caries Res 40:186-193

17. Krause F, Braun A, Frentzen M, Jepsen S (2008) Effects of composite fissure sealants on IR laser fluorescence measurements. Lasers Med Sci 23:133-139

18. Takamori K, Hokari N, Okumura Y, Watanabe S (2001) Detection of occlusal caries under sealants by use of a laser fluorescence system. J Clin Laser Med Surg 19:267-271

19. Gostanian HV, Shey Z, Kasinathan C, Caceda J, Janal MN (2006) An in vitro evaluation of the effect of sealant characteristics on laser fluorescence for caries detection. Pediatr Dent 28:445-450

20. Celiberti P, Lussi A (2007) Penetration ability and microleakage of a fissure sealant applied on artificial and natural enamel fissure caries. J Dent 35:59-67

21. Francescut P, Zimmerli B, Lussi A (2006) Influence of different storage methods on laser fluorescence values: A two-year study. Caries Res 40:181-185

22. Deery C, Fyffe H, Nugent Z, Nuttall N, Pitts N (1995) The effect of placing a clear pit and fissure sealant on the validity and reproducibility of occlusal caries diagnosis. Caries Res 29:377-381

23. Carvalho JC, Ekstrand KR, Thylstrup A (1989) Dental plaque and caries on occlusal surfaces of first permanent molars in relation to stage of eruption. J Dent Res 68:773-779

24. Pearce EIF, Larsen M, Coote G (1999) Fluoride in enamel lining pits and fissures of the occlusal groove-fossa system in human molar teeth. Caries Res 33:196-205

25. Rock WP, Weatherill S, Anderson RJ (1990) Retention of three fissure sealants resins. The effects of etching agent and curing method. Results over 3 years. Br Dent J 168:232-325 